\title{
AN APPROACH FOR SEMI-AUTOMATIC REDESIGN OF MECHATRONIC PRODUCTS BY MULTIDISCIPLINARY OPTIMISATION
}

\author{
Walter, Leonie; \\ Schleich, Benjamin; \\ Wartzack, Sandro \\ Friedrich-Alexander-Universität Erlangen-Nürnberg
}

\begin{abstract}
Mechatronic products are widely spread today, but their development still is a challenge. In addition, with the trend of individualisation, not only one solution must be designed but multiple configurations are needed to fulfil customer-specific requirements. To reduce cost and development time, usually existing products are adapted for this purpose. Even though a lot of knowledge is already available through the previous product version, because of multiple disciplines that must be considered in mechatronic systems, even redesign processes are complex. To support the adaption of mechatronic products to changed requirements, an approach to systematically reuse the knowledge available from previous product versions is proposed in this contribution. Through multidisciplinary behaviour optimisation, the solution space is reduced to build an adapted product meeting the new requirements. The approach is explained in detail and illustrated with the example of an electric window regulator and the results are discussed thoroughly.
\end{abstract}

Keywords: Mechatronics, Optimisation, Product modelling / models, Redesign

\section{Contact:}

Walter, Leonie

Friedrich-Alexander-Universität Erlangen-Nürnberg

Engineering Design

Germany

1.walter@mfk.fau.de 


\section{INTRODUCTION}

Mechatronic products are widely spread today. However, as advantages can be obtained from combining multiple disciplines, such as mechanics, electrics, and informatics, in one product, complexity in design increases also (Torry-Smith et al., 2013). In addition, there is a trend for customers to define their own specific product requirements which leads to an increasing need for individualisation in design (Lasi et al., 2014). To reduce costs and development time, previous products are usually used as basis for redesign or adaption processes (Naefe and Luderich, 2016). Developing adapted products or creating variants are the most frequent tasks in product design (Pahl et al., 2007). This leads to many similar but slightly different products that fulfil individual requirements.

Nevertheless, even though no completely new product has to be developed, adapting an existing one for new requirements still is a challenging task. Among other aspects this is because even though the main goal of a mechatronic product most likely is to fulfil a requested function, other goals must not be neglected, especially and for example costs (Ehrlenspiel and Meerkamm, 2017) or the reduction of unintended effects. Therefore, multiple optimisation goals are to be considered in adapted product development. As the system behaviour usually cannot be obtained from observing its components separately (Ropohl, 2009), the developer needs to evaluate the whole system to check whether the adapted product meets the requirements. Following the classic product development processes as described in (VDI 2206, 2004), this is done after domain-specific design. If the requirements are not met, changes must be made within the disciplines, the product assembled again, and the overall system tested once more. This leads to multiple iterations and in consequence to long development time.

However, iterations usually cannot be skipped, as a system's behaviour is determined by its properties, which mostly cannot be manipulated easily or directly by the developer. The product, however, is built by defining its characteristics (Weber and Deubel, 2003). Identifying the needed characteristics which realise the required properties is a difficult task and there still is a lack of methodical approaches to support the generation of optimal solutions in the redesign process. Nevertheless, a lot of information about the previous product version is available which can simplify purposive changes if used thoroughly. In this context, the main research challenge is to develop an approach to optimise the multidisciplinary system behaviour of mechatronic products as early as possible to reduce the solutions space and therefore to reduce the number of iterations in later design stages by systematically reusing available knowledge.

This paper is organised as follows: In section 2, the state of the art as well as related work is presented. The approach for semi-automatic multidisciplinary optimisation in mechatronic product redesign is described in general and illustrated via an example in section 3. In section 4 follows a thorough evaluation of the presented approach. The contribution is concluded and an outlook given in section 5 .

\section{RELATED WORK AND STATE OF THE ART}

One main goal of product design is to develop a system, whose behaviour fulfils a required function (Gero, 1990). Behaviour is a rather general term. This paper follows the definitions of Gero and Kannengiesser (2004) where "function" describes the products purpose, "behaviour" what the system does and "structure" the product itself. Behaviour can be distinguished in expected behaviour, which is developed from function, and the actual behaviour of the developed system. An interesting aspect is mentioned in (Ropohl, 2009): A system has attributes that contribute to the desired function but also attributes which cause secondary effects that must be considered in the development process regardless. As system design is a difficult task, systematic approaches for engineering design were developed, such as presented by Pahl et al. (2007) or as stated in (VDI 2221 Part 1, 2019). An approach especially for mechatronic products is the well-known V-model, which is described in (VDI 2206, 2004). A special procedure was necessary as in mechatronic products, the disciplines mechanical engineering, electrical engineering, and information technology are combined (VDI 2206, 2004), leading to additional difficulties, because multiple disciplines must be regarded in the design process.

As stated in the introduction, this paper focuses on redesigning mechatronic products. Redesign can be defined as the adaption of an existing product to changed requirements (VDI 2222 Blatt 1, 1997). There is no constraint whether only parameters or whole subsystems must be adapted or completely renewed. Following Naefe and Luderich (2016) the principle solution remains the same. In research there are various approaches regarding the support of changing, redesigning or adapting products. A first step to facilitate changes is to design the product right from the start in a way that enables adaptions as 
proposed by Fricke and Schulz (2005). Several contributions address the challenge of detecting and tracing change propagation throughout the redesigned system. A tool to automatically generate redesign plans to adapt a limited number of parameters based on an influence model is presented in (Ollinger and Stahovich, 2004). However, it is only identified which parameters should be increased or decreased, a specific value is not calculated.

Information management and reuse is one possibility to facilitate product development. Ahmad et al. (2013) proposed a tool to model and manage knowledge needed to understand change propagation and to predict consequences to support the designer. An approach to support change impact analysis based on the CPM/PDD-model is discussed in (Köhler et al., 2008). In (Koh et al., 2012) a method to assess the impact of change propagation against requirements is introduced. Dworschak et al. (2019) developed a method to link requirements and CAD models to automate the adaption to changed requirements. In (Dohr et al., 2014) the development of software support to connect requirements, system models and system simulation is described.

Multidisciplinary design optimisation (MDO) is a vast field of research and only a few contributions with relevant topics are listed here. Gamage et al. (2012) describe the optimisation of a mechatronic system after identifying a component needing improvement using genetic programming. Considering multiple disciplines as well as control design are general challenges in engineering design and topic of several research projects, e.g. the simulation-based multi-objective optimisation including the plant as well as the controller conducted in (Casner et al., 2012). In (Tarkian et al., 2012) an automated multidisciplinary optimisation of a robot is executed combining FE-analysis and dynamic simulation, while Tian and Voskuijl (2015) propose a framework to automatically generate multi-physics simulation models for optimisation. Mechatronic system optimisation in early design phases with limited knowledge is the subject of (Rahimi et al., 2017). Ashhab et al. (2014) introduce another method for early phases: The optimal input parameters for a manufacturing process to create a desired part can be determined with an artificial neuronal network, avoiding time-consuming simulations.

The publications presented here can only give an excerpt of important aspects, challenges and solution approaches regarding the design and redesign of mechatronic systems. Focusing on adapting already existent products, common problems are to identify the right component to change, to detect the impacts on the system and to determine the way to realise the adaption. The first two aspects of redesign are addressed in several contributions; however, there is a lack of methods to actually identify the quality of required changes, i.e. to calculate feasible values for components that need adaption.

This contribution focuses on this last aspect. With the trend to individualise products, a lot of systems only need minor changes to fulfil new requirements and great parts of a previous product version can be reused. However, with problems as the ambivalence of behaviour and structure or multidisciplinarity, the adaption is a challenging task. Therefore, in this contribution, we propose a semiautomatic approach to support the redesign process for mechatronic products. The approach is described in detail within the next chapter. Initially, an overview of the complete approach is given. A more detailed description of the different steps, models, and information needed follows afterwards. An example accompanies the explanations for better understanding.

\section{AN APPROACH FOR SEMI-AUTOMATIC REDESIGN OF MECHATRONIC PRODUCTS BY MULTIDISCIPLINARY OPTIMISATION}

An overview of the approach is depicted schematically in Figure 1 and will be described in general in this passage and more detailed in the following sections. The approach is structured corresponding to the three intermediate results, marked with (1), (2), (3) and the steps leading to these results marked with (x.1) and so forth. Starting point but not part of the approach is a fully elaborated product that shall be adapted. The models and information available from this and its development process are used to build or - if already existent - extend a knowledge base (0). The new requirements are integrated in the knowledge base as well. Additionally, the final system model is copied and used as basis for the changes to the product, which will occur later in the process. It is marked with (0). Using information from the previous system and the knowledge base, relevant aspects of the multidisciplinary system behaviour are modelled as independently from structure as possible (1.1). A connection between the behaviour and the structure model must be set up to establish a relation (1.2). Result of these steps is the multidisciplinary behaviour model (1). The next step is to set up the optimisation process (2.1) using the new requirements as goals and restrictions. Knowledge from the previous product, catalogues, experimental data and so on 
is added to limit the optimisation process (2.2) to create feasible results. These form a pareto front of possible combinations (2) which fulfil the requirements and are optimised according to specific demands. To implement the results, one possible combination is selected (3.1). Using the connection between the behaviour and the structure model, the components to change are identified (3.2). With help from the knowledge base possible changes to realise the optimised parameters (3.3) can be found and the adaption can be implemented (3.4), resulting in an adapted structure model (3) that fulfils the requirements regarded in the optimisation optimally. If the required properties could not be realised in the synthesis step or if requirements that could not be considered in the optimisation process are not met later, another solution must be selected from the pareto front (4).

This approach is explained in detail in the following sections. It is divided into the three steps "modelling the multidisciplinary behaviour", "optimisation", and "result integration". To allow for better understanding, an illustration via a persistent use case follows the general description of each step: the adaption of an electric window regulator, as it is used within a car. The initial situation and with it the knowledge base though need to be focused on in detail first.

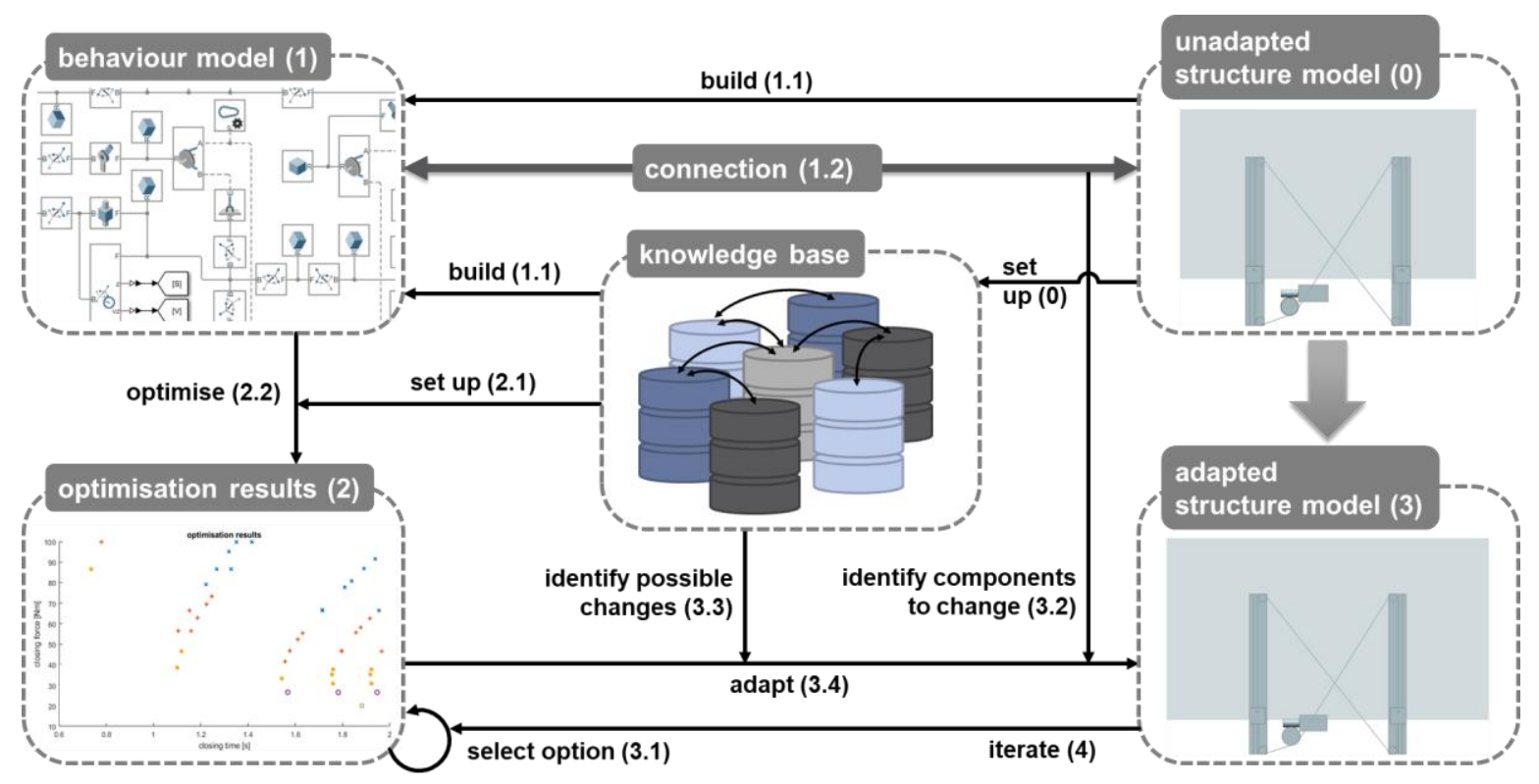

Figure 1. Overview of the approach

\subsection{Initial situation and knowledge base}

\subsubsection{General description}

The approach introduced here requires a lot of data and knowledge. Preparing the knowledge base is not explicitly part of this approach and therefore only partly represented in Figure 1, but as it is a very important aspect, it is discussed here. Starting point is a fully elaborated system that shall be adapted to changing requirements in the future. First, information about the previous design process is needed. This is especially the structure model - the singular form also includes the possibility, that there are multiple models representing this aspect - that is used as basis for the adaption and the previous requirements. The term behaviour is used here to describe what a system does and structure what it is, following the definitions of Gero and Kannengiesser (2004). How these aspects are modelled or documented is not concretised here and can differ. Helpful for setting up and verifying the behaviour model - if it is not already available from the product development process - is additional data on the system and its components like parameters or experimental results. For the optimisation and result integration, knowledge of the design process is beneficial. This could be for example decisions that lead to a certain shape, the selection of a component from a catalogue, or problems that occurred before. Information not regarded in the previous system and its design process but available within the company complements the knowledge base. This is e. g. components listed in catalogues as purchased parts or standard components that cannot be changed, available manufacturing possibilities influencing the design, or common knowledge like design rules or physical laws. In addition, it is essential for the adaption process to include the new requirements. Connecting them to the previous requirements helps 
to set up the optimisation process. How this data and knowledge is managed concretely depends mainly on the company, whether they already have a knowledge management system or not, and how the following approach will be implemented, but also on the kind of information included, its amount and other aspects. More details on the contents of the knowledge base are given when needed in the next steps, but first the use case used to illustrate this approach is introduced.

\subsubsection{Use case: presentation}

As already stated above, the example considered here is an electric window regulator for cars. A photograph and a schematic sketch are shown in Figure 2. After the window regulator has been designed for a small car it shall be adapted to fit for a bigger vehicle. The main requirements are to lift a window heavier than before but within the same time of $2 \mathrm{~s}$. Additionally, the closing force should not exceed a given value for pinch protection. The requirements are saved within a spreadsheet. A physical demonstrator is available and used to derive realistic system values, which are used to set up the behaviour model and optimisation later. At this point of time, the knowledge base only contains relevant parameters for possible alternatives of motor, worm gear and cable drum, also saved as a spreadsheet.
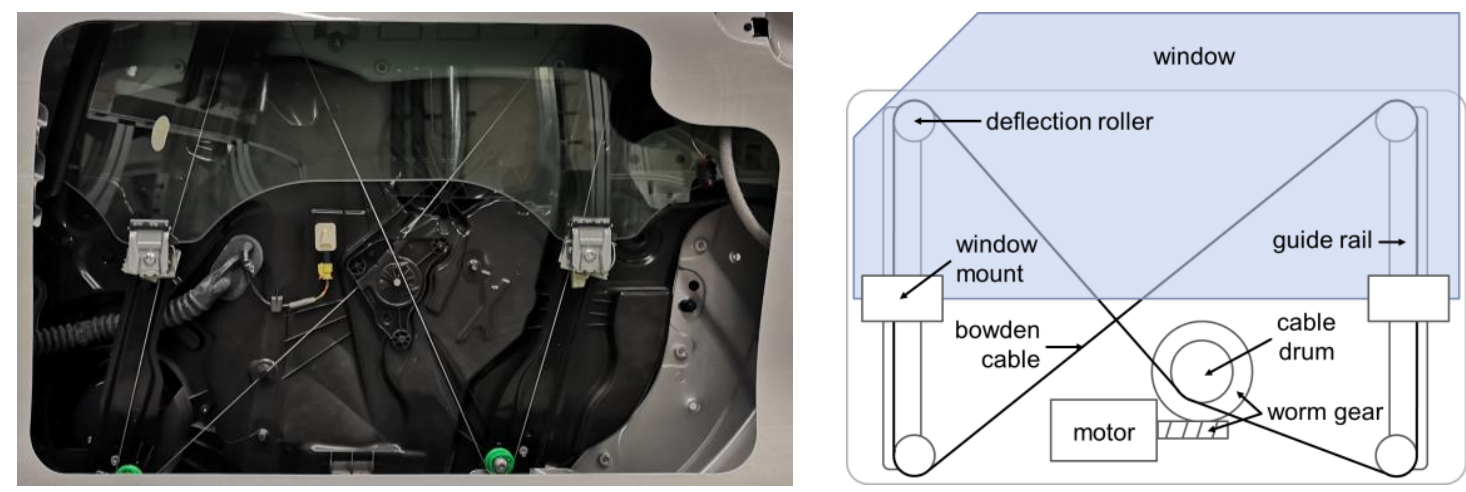

Figure 2. Photograph and schematic sketch of the demonstrator

\subsection{Multidisciplinary behaviour model}

\subsubsection{General description}

Depending on the system, its purpose, its environment, its requirements and so on, different types of behaviour are focused on within the development process. In an industrial robot for example, the multibody and drive behaviour is needed to enable movement. However, in addition the stiffness is essential for the robot to work and needs to be ensured, even though it only contributes implicitly to the required function. Electromagnetic compatibility might be not that important. This distribution however could be completely different for example in medical devices. The challenging part in multidisciplinary behaviour modelling and optimisation is, that different types of behaviour must be evaluated using various independent and specialised tools and models. The term behaviour model here - as structure model above - includes, that system behaviour can be represented not only by one model but also by multiple connected models. In this approach, the aspects most relevant for fulfilling the requirements should be focused on to avoid unnecessary effort. Through behaviour optimisation in advance to detailed design, the solution space for system synthesis shall be restricted. To avoid unnecessary limitations, system properties should be modelled as independently as possible from the characteristics.

The first task is to build a behaviour model suitable for the optimisation process. If a model already exists, it might have to be adapted to achieve this. For building a new model, the structure of the behaviour model is set up as starting point. It will stay constant throughout the adaption processes. Then, the parameters of the previous product version are used to fill the model. For the optimisation process it is necessary to identify the relevant variables, that can be adapted. To provide realistic results, their possible values should be restricted to feasible ones. This could be a continuous range or discrete values, for example for catalogue components. Parameters depending on others should be linked to avoid impossible combinations, e.g. armature resistance and inductance in a motor. The values are provided via the knowledge base. They can be imported once and updated at regular intervals or a direct link can be established between parameters and the knowledge base to always provide the current values. 
The next logical step would be to set up the optimisation process. However, to be able to adapt the structure model according to the optimisation results later in this approach, a link between them is necessary. As the behaviour and structure model represent aspects of the same system and are both constant in their structure independently from adaptions, a connection should be established between them. This can be implemented in different ways, for example it could be defined, which components belong to each other. Another option is to connect parameters to components or other parameters. Relationships and connections within the models should also be represented. As this step is tightly connected to modelling, it is executed at this point of the approach. Modelling and connecting the behaviour model requires a lot of effort and time, but only needs to be done once for all adaption processes in the future.

\subsubsection{Use case: building the behaviour model}

For the electric window regulator, the physical and the drive behaviour are very important and can be considered partially independent from the realising structure. The focussed dynamic system behaviour is modelled in Matlab/Simulink using the Simscape library. This program has been selected instead of Modelica and other similar tools respectively languages because of its connection to Matlab, which can be used for optimisation. The model is structured to represent the modular composition of the window regulator. The mechanical parts are mainly described by multibody components with a link to an electromechanic motor. This model and an extract of it are shown in Figure 3. The structure and values are derived as far as possible from a physical example. The values are set parametrically using a script.
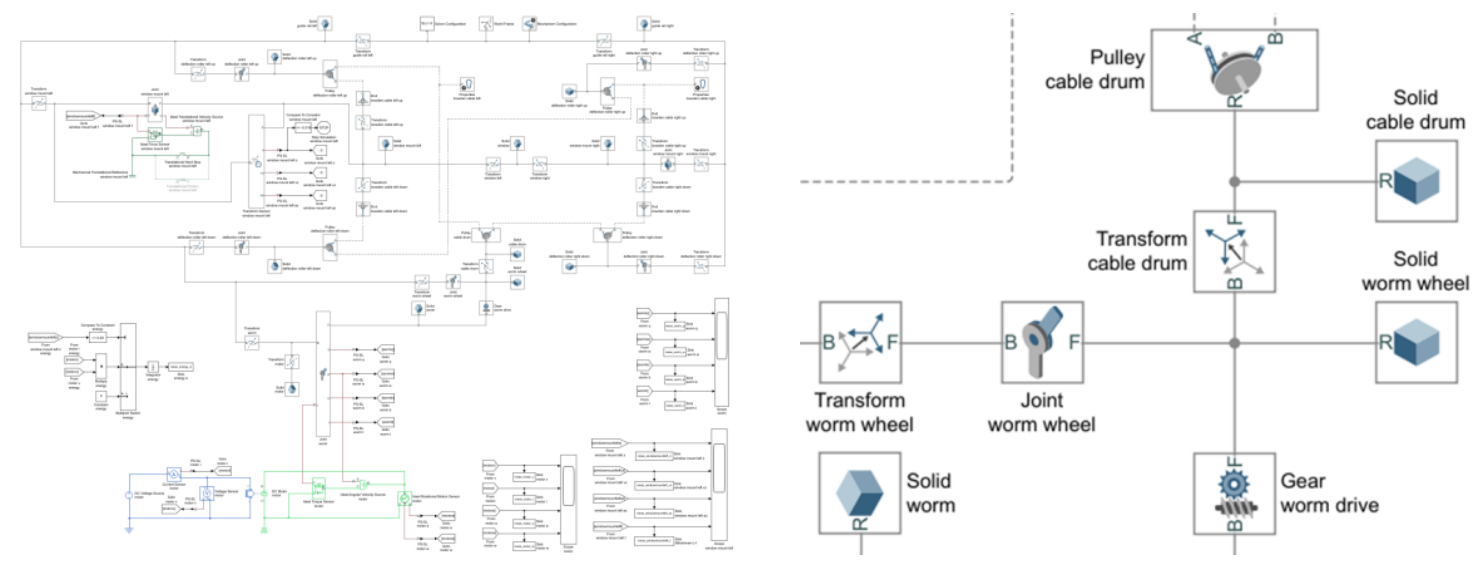

Figure 3. Simulink model of the electric window regulator (complete and extract)

\subsection{Optimisation process for redesign}

\subsubsection{General description}

To optimise the behaviour, the optimisation process must be set up. First, the new requirements must be considered. They define the optimisation goal and may pose additional restrictions and therefore must be included in the optimisation algorithm. Possibly different application scenarios of the same system must be determined. The next step is to identify the parameters to adapt in the optimisation process. Possible values to consider are gathered from the knowledge base. Additionally, the technical aspects of the optimisation process must be defined, i.e. the optimisation algorithm and depending on this e.g. simulation time or number of iterations. Depending on the type of the behaviour model, different approaches are possible. If equations are available, direct calculations are possible. For a simulation model, an iterative procedure between simulation and result evaluation can be applied. The desired outcome of the optimisation process are system configurations capable to fulfil the required goals and restrictions. They form the resulting pareto front. As in step (1), setting up the behaviour model as well as identifying and implementing a suitable optimisation process only needs to be done once for the initial design of a new system or for setting up this approach for an existing product.

\subsubsection{Use case: redesign by optimisation}

In this use case, the window regulator shall be adapted for a bigger car. The window size changes from $700 \times 400 \times 4 \mathrm{~mm}$ to $800 \times 450 \times 4 \mathrm{~mm}$. The requirement to close the window within two seconds stays the 
same. The maximum closing force is reduced from $150 \mathrm{~N}$ to $100 \mathrm{~N}$. These are selected as the optimisation goals for the closing procedure. The optimisation process is realised as Matlab script. The components to change and their possible values are selected. Here, only the motor, worm gear and cable drum are changed. For each motor and gear, seven possible options within a certain range of the previous parameters are selected. The motor stall torque ranges from $0.05 \mathrm{Nm}$ to $0.2 \mathrm{Nm}$ and the idle speed from $30001 / \mathrm{min}$ to $40001 / \mathrm{min}$. The original translation ratio of the gear was 40 and is now limited to a range from 30 to 90 in steps of 10 . The cable drum's radius was $35 \mathrm{~mm}$ and can be changed in steps of $5 \mathrm{~mm}$, with a minimum of $30 \mathrm{~mm}$ and a maximum of $50 \mathrm{~mm}$. The target is, to find all combinations of these components that fulfil the required time and force. In the optimisation algorithm, all possible combinations of motor, gear and cable drum are considered successively. For each parameter combination, the closing process is simulated and the necessary closing time and force are measured. If a combination is found fulfilling both requirements, it is saved as a possible result. Additionally, the electrical energy needed to close the window is measured. It is explicitly not included in the optimisation to provide multiple solutions, in case that the optimal combination cannot be realised physically. The resulting pareto front is shown in Figure 4. The next step is to adapt the CAD model according to one solution out of these results.

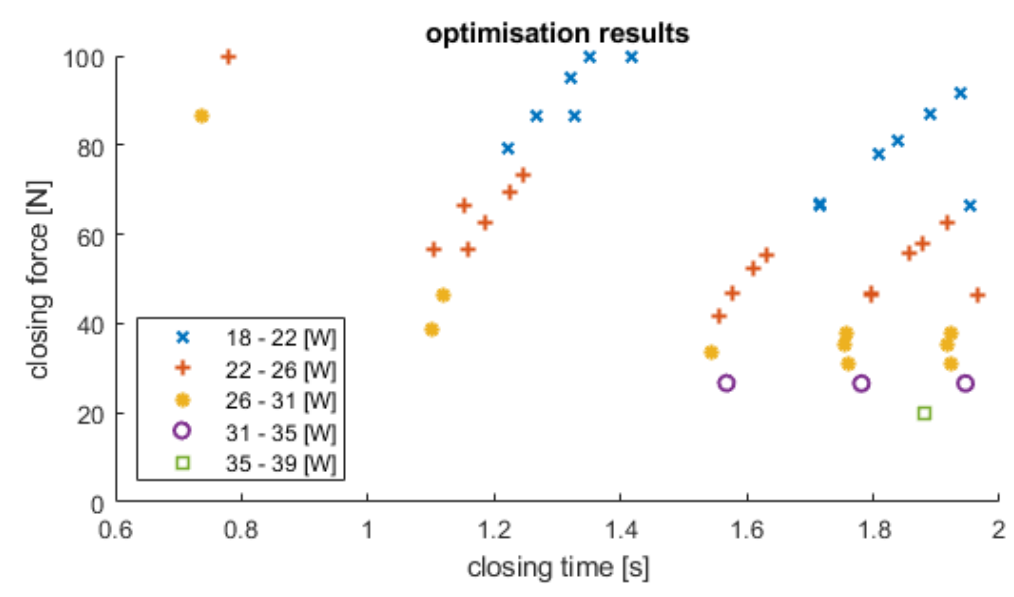

Figure 4. Pareto front resulting from the optimisation process

\subsection{Result integration}

\subsubsection{General description}

As possible property combinations have been identified, the system's characteristics must be adapted. In order to do this, the first task is to select one parameter combination from the results. This could be done following different criteria, but a reasonable option is to select a combination with the least difference to the previous system. Another one could be to reduce the number of parts that need to be adapted. More elaborate would be to rate different parameters on how easily they can be changed and if this causes necessary changes in other components. Independently on how the combination is selected, it must be determined which parts must be adapted. This is done using the connection between the behaviour and the structure model. A comparison of previous values helps to identify which aspects of the component must be changed. Here should be considered, that similar properties can be realised by different characteristics, so that parts that primarily did not need changing can or must be adapted if necessary. How the adaption can be implemented depends mainly on the component. If the part itself is not changeable as e.g. purchased parts, a suitable replacement must be selected from the knowledge base. If the component itself is changeable, adaptions can be made. Depending on the manner it is modelled there are different ways. When there is a clear connection between properties and characteristics, for example through equations or rules, this might be done automatically. With more complex relations, more elaborated approaches might be able to find a solution, but some changes might require the expertise of a developer. After the type of change has been identified, it should be applied to the structure model. Here problems can arise, for example if not enough space is available or interfaces are not compatible. If this is the case, a different kind of change should be selected.

If the adaption was successful, the redesigned system should fulfil the requirements included in the optimisation process. However, aspects that could not be considered there, for example because they 
depended on the detailed structure, must be evaluated now to guarantee product functionality. If they cannot be fulfilled, adaptions are necessary through iterations to any arbitrary previous sub-step within the result integration process, from selecting another option from the pareto front to adapting the system.

\subsubsection{Use case: selecting a redesign option and adapting the CAD model}

For this use case, the solution requiring the least energy was picked from the pareto front; marked with a red " $x$ " in Figure 5. For this all three regarded parts - i.e. motor, worm gear and cable drum - change, the cable drum radius from 35 to 40 , the gear ratio from 40 to 50 and the motor stall torque from $0.15 \mathrm{Nm}$ to $0.1 \mathrm{Nm}$ while its idle speed remains constant with $3000 \mathrm{1} / \mathrm{min}$. The window regulator's physical structure is modelled parametrically in Siemens NX. To adapt this model, the geometrical parameters of the relevant components are retrieved from the knowledge base and automatically changed in NX with a Visual Basic script. Not evaluated until now are aspects as weight, cost, or acceptable noise. However, the iterations between detailed design and evaluation of the closing time as one essential requirement could be shifted from system to component level. As the original and changed CAD models look very similar (see Figure 1), the changed parameters are displayed in Figure 5.

Another possible way to select a solution as mentioned above is to reduce the number of components to exchange. Figure 5 also shows, that the previous window regulator could not fulfil the new requirements, but there are five options, where only one part, and several more, where only two components have to be adapted. This could be a better choice, if there are existing contracts with suppliers for some parts.

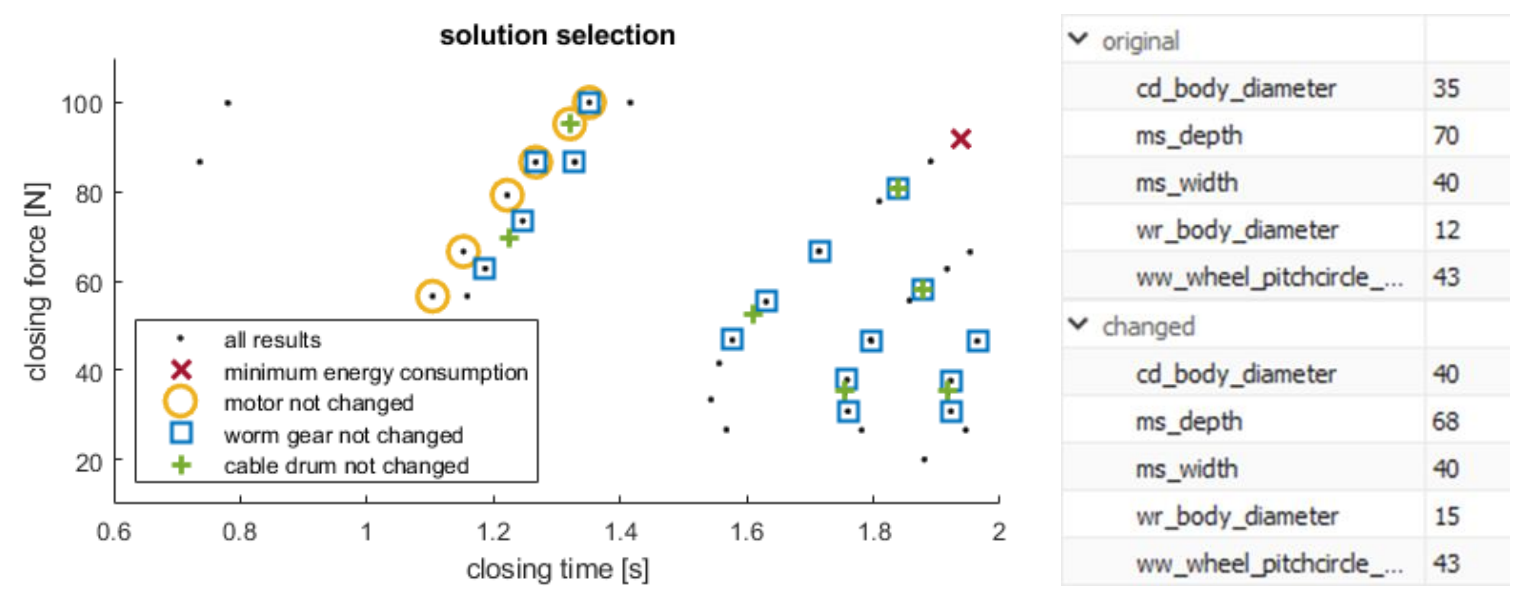

Figure 5. Some solutions selected following different criteria and changed parameters in Siemens NX

\section{DIscussion}

The case study illustrated that the redesign of a mechatronic product by behaviour optimisation following the proposed approach works well and returns reasonable results. Even though there is room for improvement, the potential benefits are recognisable.

\subsection{Limitations and challenges}

One drawback at the moment is the simple parametric support for integrating the obtained results into the structure model. Different, more elaborate approaches e.g. for modular components or parts based on design rules are possible, but their functionality has not been tested yet.

Another challenge is, that for the integration step as well as the optimisation process itself and the modelling an enormous amount of data and knowledge is necessary. First, the needed information must be gathered from different sources as the previous product, experiments, developer knowledge, suppliers and so on. Second, the collected data needs to be managed, which is challenging due to the various types of data, the need to access it at different stages of the approach, the need to keep it up to date and more.

Even if enough information is available, building a behaviour model suitable for the optimisation process is difficult, especially for this approach as properties and characteristics should be separated and realistic values need to be predicted. Additionally, the model should be as precise as possible to obtain reasonable results but also preferably simple to reduce optimisation effort. Even though the approach is rather general, it should be concretised what a suitable model is. 
The optimisation process is executed using a full-factorial algorithm considering only discrete values based on the system simulation. Calculating all possible combinations and evaluating them afterwards only works for very small problems and there is a need to select a more suitable algorithm in order to reduce time and improve the results. However, in this contribution the optimisation algorithm was not focused on yet, as the main goal was to show the overall approach to optimise the behaviour before detailing the form. As indicated in the related work chapter, there is a vast amount of various approaches and algorithms to optimise multidisciplinary system behaviour. Even though a thorough analysis is yet necessary to find a suitable optimisation algorithm for this approach, this should be possible.

\subsection{Advantages and benefits}

Summarising the evaluation up to now, there are multiple challenges that must be addressed, however, several of them also appear in conventional mechatronic design and redesign processes. One example is the vast amount of information needed for the knowledge base. Great parts of this would also be required for a manual redesign process. Actively managing knowledge and data as it is done in the presented approach provides numerous advantages. The knowledge gained within the development of previous product versions can be stored and reused, saving the time needed to search for it or even to repeat parts of the development. In addition, with an elaborated knowledge base, even non-productspecific knowledge can be managed and easily integrated in the adaption process. Gathering the required knowledge focuses on the previous product knowledge and therefore should presumably not be extraordinary difficult and time-consuming.

At first glance the amount of time required not only to set up the knowledge base but also the whole process is a major drawback. Though effort is needed for the preparation of the first redesign process, any number of adaptions are possible afterwards, saving time with every required adaption. Comparing the presented to a manual redesign process, developers have more time for other assignments through the automation of non-creative standard tasks. Time can also be saved through the early determination of requirements for the domain specific design. Iterations to evaluate certain aspects of behaviour can be avoided on system level, as they can be proven for the components separately. The restriction of the solutions space prevents waste of time on the elaboration of elements that do not fulfil given requirements.

This leads to a more purposeful procedure to develop an optimal product. For the optimisation process, multiple objectives can be considered and rated corresponding to their importance. They can be changed easily depending on the current situation. The identified requirements for domain specific design not only lead to fewer time needed for iterations but may also support the developer for the detailed design process by providing clear goals and limitations. On system level, the presented approach enables a purposeful and systematic generation of optimal products regarding the defined goals.

\section{CONCLUSION}

In this contribution, an approach to reduce iterations in redesign of mechatronic products through multidisciplinary behaviour optimisation and the reduction of solution space has been proposed. The necessary steps and information have been explained and proven with an electric window regulator as example. Moreover, advantages of this approach and future challenges have been discussed.

For future work, various parts of the approach should be enhanced and extended. The next step is to improve the optimisation process, to consider not only discrete but also continuous variables and to use a more suited and efficient algorithm. Additionally, setting up the approach increasingly modular would elevate its reusability and flexibility for more than parametric changes and alternative solution concepts. However, the approach is a first step towards the automatic redesign of mechatronic products.

\section{ACKNOWLEDGEMENTS}

This research work is part of the FAU "Optimization-based design methodology in early phase of mechatronic product development" project (EFRE/OptMePro) and funded by the Bavarian program for the "Investment for growth and jobs" objective finance by the European Regional Development Fund (ERDF), 2014-2020. The authors are responsible for the content of this publication. 


\section{REFERENCES}

Ahmad, N., Wynn, D.C. and Clarkson, P.J. (2013), "Change impact on a product and its redesign process: A tool for knowledge capture and reuse", Research in Engineering Design, Vol. 24 No. 3, pp. 219-244. https://dx.doi.org/10.1007/s00163-012-0139-8.

Ashhab, M.S., Breitsprecher, T. and Wartzack, S. (2014), "Neural network based modeling and optimization of deep drawing - Extrusion combined process", Journal of Intelligent Manufacturing, Vol. 25 No. 1, pp. 77-84. https://dx.doi.org/10.1007/s10845-012-0676-z.

Casner, D., Renaud, J. and Knittel, D. (2012), "Computer-aided design of mechatronic systems using multiobjective optimization and object-oriented languages”, ASME 2012 11th Biennial Conference on Engineering Systems Design and Analysis, ESDA 2012, pp. 301-310. https://dx.doi.org/10.1115/ESDA2012-82212.

Dohr, F., Eisenbart, B., Huwig, C., Blessing, L. and Vielhaber, M. (2014), "Software support for the consistent transition from requirements to functional modeling to system simulation", 10th Biannual NordDesign Conference, NordDesign 2014, pp. 775-784. ISBN 978-190467058-2.

Dworschak, F., Zirngibl, C., Schleich, B. and Wartzack, S. (2019), "Konzept für den MBSE-Einsatz zur automatisierten Individualisierung von komplexen Produkten”, 30th Symposium Design for X, DFX 2019 , pp. 279-290.

Ehrlenspiel, K. and Meerkamm, H. (2017), Integrierte Produktentwicklung, Carl Hanser Verlag, München. ISBN 978-3-446-44908-4.

Fricke, E. and Schulz, A.P. (2005), "Design for changeability (DfC): Principles to enable changes in systems throughout their entire lifecycle", Systems Engineering, Vol. 8 No. 4, pp. 342-359. https://dx.doi.org/10.1002/sys.20039.

Gamage, L.B., De Silva, C.W. and Campos, R. (2012), "Design evolution of mechatronic systems through modeling, on-line monitoring, and evolutionary optimization”, Mechatronics, Vol. 22 No. 1, pp. 83-94. https://dx.doi.org/10.1016/j.mechatronics.2011.11.012.

Gero, J.S. (1990), “Design prototypes. A knowledge representation schema for design”, AI Magazine, Vol. 11 No. 4, pp. 26-36. ISSN 07384602.

Gero, J.S. and Kannengiesser, U. (2004), "The situated function-behaviour-structure framework", Design Studies, Vol. 25 No. 4, pp. 373-391. https://dx.doi.org/10.1016/j.destud.2003.10.010

Koh, E.C.Y., Caldwell, N.H.M. and Clarkson, P.J. (2012), "A method to assess the effects of engineering change propagation", Research in Engineering Design, Vol. 23 No. 4, pp. 329-351. https://dx.doi.org/10.1007/s00163-012-0131-3.

Köhler, C., Conrad, J., Wanke, S. and Weber, C. (2008), “A matrix representation of the CPM/PDD approach as a means for change impact analysis", 10th International Design Conference, DESIGN 2008, pp. 167-174. ISBN 978-953631389-1

Lasi, H., Fettke, P., Kemper, H.-G., Feld, T. and Hoffmann, M. (2014), "Industry 4.0”, Business and Information Systems Engineering, Vol. 6 No. 4, pp. 239-242. https://dx.doi.org/10.1007/s12599-014-0334-4

Naefe, P. and Luderich, J. (2016), Konstruktionsmethodik für die Praxis, Springer Fachmedien, Wiesbaden. ISBN 978-3-658-13871-4.

Ollinger, G.A. and Stahovich, T.F. (2004), "RedesignIT - A model-based tool for managing design changes", Journal of Mechanical Design, Vol. 126 No. 2, pp. 208-216. https://dx.doi.org/10.1115/1.1666888.

Pahl, G., Beitz, W., Feldusen, J. and Grote, K.-H. (2007), Pahl/Beitz Konstruktionslehre, Springer-Verlag, Berlin. ISBN-13 978-3-540-34060-7.

Rahimi, F., Genf, L., Wikander, J. and Frede, D. (2017), "Early phase design-optimization of mechatronic systems", 5th International Conference on Control, Mechatronics and Automation, ICCMA 2017, pp. 42-49. https://dx.doi.org/10.1145/3149827.3149838.

Ropohl, G. (2009), Allgemeine Technologie, Universitätsverlag Karlsruhe, Karlsruhe. ISBN 978-3-86644-374-7.

Tarkian, M., Persson, J., Ölvander, J. and Feng, X. (2012), "Multidisciplinary design optimization of modular industrial robots by utilizing high level CAD templates", Journal of Mechanical Design, Vol. 134 No.12. https://dx.doi.org/10.1115/1.4007697.

Tian, F. and Voskuijl, M. (2015), "Automated generation of multiphysics simulation models to support multidisciplinary design optimization”, Advanced Engineering Informatics, Vol. 29 No.4, pp. 1110-1125. https://dx.doi.org/10.1016/j.aei.2015.07.004.

Torry-Smith, J.M., Qamar, A., Achiche, S., Wikander, J., Mortensen, N.H. and During, C. (2013), "Challenges in Designing Mechatronic Systems”, Journal of Mechanical Design, Vol. 135 No. 1. https://dx.doi.org/10.1115/1.4007929.

VDI 2206 (2004), Design methodology for mechatronic systems, Beuth Verlag GmbH, Berlin.

VDI 2221 Part 1 (2019), Design of technical products and systems - Model of product design, Beuth Verlag $\mathrm{GmbH}$, Berlin.

VDI 2222 Blatt 1 (1997), Methodic development of Solution principles, Beuth Verlag GmbH, Berlin.

Weber, C. and Deubel, T. (2003), "New theory-based concepts for PDM and PLM", 14th International Conference on Engineering Design, ICED 2003. ISBN 1904670008. 